\title{
Tecnura
}

\section{Evaluación de impacto ambiental de la cantera "La Represa", en la provincia de San Luis, Argentina}

\author{
Environmental impact assessment of the quarry "La Represa" \\ in San Luis province, Argentina
}

\author{
Natalia Judith Marchevsky ${ }^{1}$, Andrea Alejandra Giubergia ${ }^{2}$ \\ Néstor Hugo Ponce ${ }^{3}$
}

Fecha de recepción: 22 de diciembre de 2017

Fecha de aceptación: 9 de marzo de 2018

Cómo citar: Marchevsky, N.J., Giubergia, A.A. y Ponce, N.H. (2018) Evaluación de impacto ambiental de la cantera "La Represa" en la provincia de San Luis, Argentina. Revista Tecnura, 22(56), 51-61. DOI: https://doi. org/10.14483/22487638.12907

\section{Resumen}

Contexto: Los estudios de impacto ambiental (EIA) constituyen herramientas importantes puesto que permiten pronosticar las consecuencias que tiene el desarrollo de una actividad extractiva en el entorno donde se localiza. El objetivo de este trabajo es adelantar la evaluación ambiental de la cantera de piedra laja La Represa, ubicada en la provincia de San Luis, Argentina.

Método: Se utilizó una matriz de doble entrada que permite estimar los impactos ambientales en el medio receptor. Dicha matriz considera determinadas cualidades de los pasivos ambientales que se introducen en una función para generar un índice denominado importancia del pasivo ambiental (IM). Mediante la comparación de estos índices es posible determinar los impactos ambientales que resultan críticos para el entorno estudiado.

Resultados: El análisis efectuado permitió determinar que el paisaje es el único elemento ambiental que tiene una afectación negativa crítica en inmediaciones de la explotación de la cantera. No obstante, se evidenció un importante mejoramiento en la calidad de vida de los pobladores de la zona a partir de este emprendimiento minero.

Conclusiones: La explotación de piedra laja es primordial para la manutención de varias familias en la localidad de Riocito. Sin embargo, es conveniente tomar medidas que puedan amortizar la afectación paisajística en el lugar. Este trabajo propone algunas opciones de remediación y restauración que podrían implementarse durante y al finalizar la operación.

Palabras clave: cantera, evaluación de impacto ambiental, piedra laja.

\begin{abstract}
Context: Environmental impact studies (EIS) are important tools since they allow forecasting the consequences of the development of an extractive activity in the environment that surrounds it. The objective of
\end{abstract}

1 Ingeniera en Minas con orientación en metalurgia, doctora en el Área de Recursos Naturales de la Universidad Nacional del Nordeste. Profesor adjunto del departamento de minería, facultad de Ciencias Físico-Matemáticas y Naturales de la Universidad Nacional de San Luis. Chacabuco 917, (5700).San Luis, Argentina. Contacto: nmarchevsky@unsl.edu.ar

2 Ingeniera en Minería, magíster en Ingeniería del Software. Profesora adjunta del departamento de minería, facultad de Ciencias Físico-Matemáticas y Naturales de la Universidad Nacional de San Luis. Chacabuco 917, (5700). San Luis, Argentina. Contacto: aagiuber@unsl.edu.ar

3 Ingeniero en Minería, profesor adjunto del departamento de minería, facultad de Ciencias Físico-Matemáticas y Naturales de la Universidad Nacional de San Luis. Chacabuco 917, (5700). San Luis, Argentina. Contacto: nhponce@unsl.edu.ar 
this work is to perform the environmental impact assessment of "La Represa," a flagstone quarry located in San Luis Province, Argentina.

Method: A double-entry matrix was used to assess the environmental impacts in the environment affected by extractive activities. This matrix considers certain qualities of environmental liabilities that are introduced in a function to generate an index caIled "Importance of Environmental Liability" (IM). By comparing these indices, it is possible to determine the environmental impacts that are critical for the studied environment.

Results: The analysis allowed to determine that the landscape is the only environmental element that has a critical negative impact in the vicinity of the exploitation of the quarry. However, there was evidence of an important improvement in the quality of life of the inhabitants of the area from this mining undertaking.

Conclusions: The exploitation of flagstone is essential for the maintenance of several families in the village of Riocito. However, it is advisable to take measures that can amortize the negative effect on the landscape of the place. This paper proposes some remediation and restoration options that could be implemented during and at the end of the operation.

Keywords: environmental impact assessment, flagstone, quarry.

\section{Introducción}

En Argentina, los emprendimientos mineros que surgen muchas veces son centro de debate por la sustentabilidad ambiental de las condiciones en las cuales se desarrollan. En consecuencia, la falta de información ambiental, la escasa o nula participación ciudadana, y la falta de transparencia en el manejo de los beneficios de la industria, sumado a los impactos negativos documentados (accidentes y derrames), han generado una percepción desfavorable de la actividad en términos ambientales (Ministerio de Ambiente y Desarrollo Sustentable, 2016).

En gran medida, el panorama descrito deriva del escaso grado de aplicación de la normativa ambiental que tiene esta actividad extractiva en el país. Los inicios de la legislación ambiental minera se remontan al año 1886, cuando se sancionó el Código de Minería de la Nación. Muchos años pasaron hasta que se implementaron nuevas medidas en torno a este tema. En 1994 se realizó la reforma de la Constitución Nacional, en la cual se incorporaron muchos instrumentos de política ambiental, como el ordenamiento ambiental del territorio, la información pública, la información ciudadana y la evaluación de impacto ambiental. En 1995, se incorporó al Código de Minería la Ley 24.585, como título complementario sobre "Protección Ambiental para la Actividad Minera". Estos dos últimos sucesos han sido significativos, dado que han instado al sector público y privado a redoblar los esfuerzos para conseguir un mejor desempeño ambiental en la actividad minera (Ministerio de Industria, Comercio, Minería y Transporte, 2014).

La Ley 24.585 establece la obligatoriedad de realizar estudios de impacto ambiental (EIA) a todo emprendimiento minero de baja, mediana o gran producción, y en cualquier etapa del ciclo minero (prospección, exploración, explotación, cierre y abandono) (Ley 24.585, 1996). Los EIA brindan información detallada de la geología, hidrogeología, vegetación, fauna, fisonomía, clima y otras características ambientales de la zona de estudio (Careddu y Siotto, 2011). Asimismo, constituyen herramientas que permiten predecir las consecuencias que tienen la ejecución y posterior desarrollo de esta actividad, en el entorno donde se localizan de modo que permiten visualizar los efectos desencadenantes $y$, en consecuencia establecer medidas preventivas y de control que hagan posible el desarrollo de esta actividad con la menor incidencia en el medio ambiente (IGME, 1999). No obstante, la legislación nacional aún presenta un 
vacío en términos metodológicos acerca de cómo deben realizarse estos EIA.

Este trabajo propone efectuar el estudio de impacto ambiental de una cantera ubicada en Riocito, donde se lleva a cabo la explotación de piedra laja pizarra. El conocimiento de esta información podría ser relevante para tomar medidas tendientes a minimizar los impactos negativos que causa la explotación de estos recursos en el ambiente.

\section{Antecedentes del lugar}

Desde mediados del siglo XX, la provincia de San Luis se distingue por el auge adquirido en materia de explotación de rocas ornamentales (lajas, granitos y mármoles). Las piedras lajas pizarras son un recurso de gran potencial y con un número significativo de pequeños productores que se localizan principalmente en Riocito. Se estima que allí existen al menos 29 canteras de piedra laja registradas (Ministerio de Industria, Comercio, Minería y Transporte, 2014), pero solo 10 se encuentran en producción. La cantera más representativa en cuanto a su producción es La Represa y ha sido seleccionada para desarrollar el presente trabajo.

\section{Ubicación y acceso}

La cantera de piedra laja pizarra La Represa se encuentra ubicada en el Partido del Totoral, departamento Pringles de la provincia de San Luis, Argentina. A este lugar se accede desde la ciudad capital de San Luis, a través de la ruta provincial No. 20 hasta la localidad de El Volcán, allí se toma la ruta provincial No. 9 hasta la bifurcación con la localidad de El Trapiche, donde se continúa algunos kilómetros más por la ruta provincial No. 39. La distancia desde la ciudad capital es de $50 \mathrm{~km}$ aproximadamente.

\section{Geología}

La cantera La Represa se encuentra emplazada sobre rocas del basamento cristalino, del cual forman parte las filitas y pizarras. Estas constituyen una faja de longitud aproximada de $50 \mathrm{~km}$, ancho variable que alcanza hasta $2 \mathrm{~km}$, dirección de aproximadamente $\mathrm{N}-\mathrm{S}$ e inclinación casi vertical.

En general, la piedra laja pizarra se compone por material clorítico-sericitico (54\%), cuarzo $(40 \%)$, calcita (3\%) y minerales opacos (3\%), de grano muy fino (Ministerio de Industria, Comercio, Minería y Transporte, 2014). Se presenta en varias tonalidades de gris, negro y verde.

\section{Ciclo de explotación de la cantera}

La Represa produce piedra laja azulina y negra, y es la cantera de mayor producción de la provincia de San Luis (5000 toneladas/mes), con reservas que superan las $300.000 \mathrm{t}$. No obstante, se estima que entre el $50 \%$ y $60 \%$ del volumen que se extrae constituye material de descarte (Ministerio de Industria, Comercio, Minería y Transporte, 2014). La figura 1 muestra una imagen de Google Earth de dicha cantera.

La zona donde está emplazado el yacimiento presenta una cubierta vegetal escasa de pocos centímetros de espesor. La operación de destape para extraer este material se realiza por medio de una topadora o cargador frontal, más voladuras de apertura de cantera. Los volúmenes extraídos de este material son acumulados sobre los sectores linderos del yacimiento de manera que no afecte la explotación futura, ni las reservas.

El laboreo del yacimiento se realiza como cantera a cielo abierto. Actualmente, tiene frentes de extracción, con bancos que superan en algunos casos los $20 \mathrm{~m}$ de altura, lo que permite tener varias alternativas de extracción. La figura 2 muestra una imagen panorámica de la cantera La Represa, se observan accesos a diferentes frentes de explotación como también material estéril acumulado.

La explotación es selectiva. Cuando el frente lo permite, se realizan perforaciones con wagon driII. En caso de aparecer venillas, vetas de otros minerales u algún otro inconveniente en el frente, se recurre a martillos neumáticos. El material se extrae por medio de voladuras con perforaciones en una malla conveniente de acuerdo con el rendimiento de los explosivos para alcanzar la máxima eficiencia. 


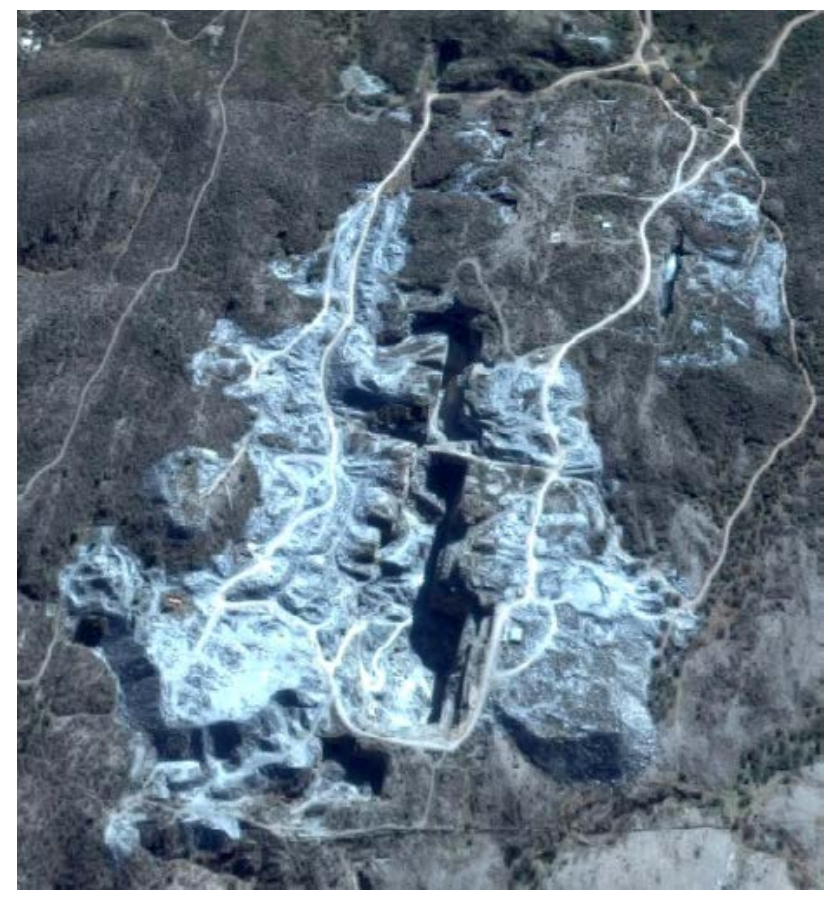

Figura 1. Vista aérea de la cantera La Represa

Fuente: google earth.

Las voladuras principales se complementan con voladuras secundarias para reducir el tamaño de los bloques grandes desprendidos del banco y que por su tamaño dificultan el proceso; en un futuro se prevé la incorporación de un martillo picador para realizar este trabajo.
En la cantera se utiliza relativamente poco equipamiento, este consiste en: cargador frontal sobre ruedas, retroexcavadora sobre orugas, compresor, martillos neumáticos, camiones con capacidad de 12 a $15 \mathrm{~m}^{3}$.

\section{Metodología}

La metodología que se utilizó en este trabajo es la descrita por García, García y Agudelo (2014), quienes han efectuado una adaptación de la propuesta por Gómez (1999). Consiste en elaborar una matriz de doble entrada, la cual identifica y caracteriza los impactos en las casillas de cruce. Según la naturaleza de los efectos causados en el medio receptor se asigna un valor de importancia al pasivo ambiental analizado. Esta metodología posibilita una estimación cualitativa y cuantitativa de los elementos, e identifica el impacto ambiental originado por una actividad específica sobre un factor ambiental.

La importancia del pasivo ambiental sobre el ambiente receptor queda determinada a través de ciertas cualidades de este, que se introducen en una función. A partir de allí, se genera un índice único denominado importancia del pasivo ambiental (IM). La ecuación (1) describe la relación entre los componentes del indicador IM.

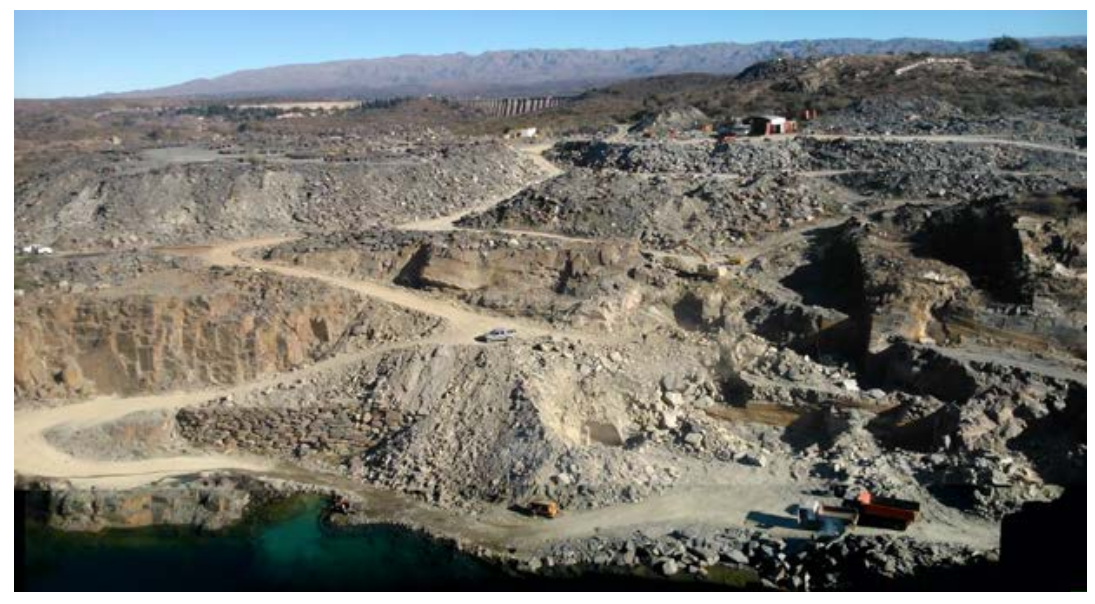

Figura 2. Foto panorámica de la cantera La Represa donde se observan accesos a diferentes frentes de explotación Fuente: Dolcemascolo (2016). 


$$
I M=N A(3 M G+2 E X+D R+P E+R C+R V+P O+T D+T I)
$$

\section{Donde:}

$N A$ : naturaleza del impacto, si este es beneficioso (+) o dañino (-).

MG: magnitud. Cuantifica el grado de incidencia. Su rango comprende valores entre 1 (afectación mínima) y 8 (afectación máxima).

EX: extensión. Calcula el área de influencia teórica del impacto respecto al medio circundante al emprendimiento. Admite valores entre 1 (efecto localizado) y 8 (efecto generalizado en todo el entorno).

$D R$ : duración. Pondera la persistencia del efecto desde su aparición y el momento en el cual el factor afectado retornaría a las condiciones originarias previas a la acción. El rango de valoración del impacto puede ser fugaz (valor 1 = duración del impacto $<1$ año) a permanente (valor $12=>$ 10 años).

$P E$ : periodicidad. Establece la regularidad de manifestación del efecto. Puede ser irregular valor $=1$ (forma impredecible) hasta continua, valor = 12 (constante a través del tiempo).

$R C$ : recuperabilidad. Se refiere a la posibilidad de retornar a las condiciones iniciales, por medio de la intervención humana. En función de cuando es posible realizar las actividades de recuperación del impacto se establece el rango de valoración que oscila entre 1 (cuando es posible realizarlo en la fase del proyecto) y 12 (cuando estas tareas no son posibles).

$R V$ : reversibilidad. Indica la posibilidad de que el factor afectado pueda regresar a las condiciones iniciales por medios naturales. Su clasificación se asigna en función del tiempo que demande este proceso; puede ser a corto plazo (valor $1=<1$ año) o irreversible (imposibilidad de retomar por medios naturales a las condiciones naturales, o hacerlo en un periodo mayor de 10 años).

$P O$ : probabilidad de ocurrencia. Describe el tiempo que acontece entre la aparición de la acción y el comienzo del efecto sobre el medio. En este caso la valoración va desde 1 (cuando el efecto demora más de 5 años en manifestarse) hasta 4 (cuando sucede en el término de 1 año). En caso que suceda algún evento crítico en el momento del impacto se añaden 4 unidades.

$T D$ : tendencia. Se refiere al aumento paulatino de la manifestación del efecto cuando la acción que lo genera persiste de forma extendida o reiterada. Se le asigna un valor $=1$ cuando el impacto es concreto y no induce nuevos efectos; o bien un valor $=2$ cuando el efecto puede prolongarse en el tiempo y aumentar paulatinamente su peligro.

TI: tipo. Establece la relación causa/efecto. Se interpreta como la forma de manifestación del efecto sobre un factor como consecuencia de una acción. Adquiere el valor $=1$ cuando la manifestación es indirecta en relación a la acción; o bien, valor = 2 cuando el efecto repercute inmediatamente en algún componente ambiental.

Un mayor detalle, así como las valoraciones intermedias de estos componentes, pueden encontrarse en el trabajo de García, García y Agudelo (2014).

Una vez obtenido el IM (ecuación 1), la importancia de un impacto, ya sea de naturaleza negativa o positiva, se determina teniendo en cuenta los criterios de calificación que se muestran en la tabla 1.

Tabla 1. Valores para clasificación de impactos (negativos/positivos)

\begin{tabular}{ll}
\hline Impactos irrelevantes & $\begin{array}{l}\text { Impactos con valores de impor- } \\
\text { tancia menor a }-25(<-25)\end{array}$ \\
\hline \multirow{2}{*}{ Impactos moderados } & $\begin{array}{l}\text { Impactos con valores de impor- } \\
\text { tancia entre }-25 \text { y menor a }-50 \\
(-25 y<-50)\end{array}$ \\
\hline Impactos severos & $\begin{array}{l}\text { Impactos con valores de impor- } \\
\text { tancia entre }-50 \text { y }-75\end{array}$ \\
\hline Impactos críticos & $\begin{array}{l}\text { Impactos con valores de impor- } \\
\text { tancia mayor a }-75(>-75)\end{array}$ \\
\hline
\end{tabular}

Fuente: elaboración propia. 


\section{Resultados y discusión}

La tabla 2 muestra los resultados de la evaluación de impacto ambiental para la cantera La Represa en fase de explotación.

\section{Componente atmosférico}

El masivo tráfico vehicular en las ciudades y los desechos generados por las industrias, como la minería, son los principales responsables de la contaminación atmosférica. Esto se debe a que ambos factores desempeñan un rol esencial en la generación de partículas y participan de manera directa e indirecta en la formación de aerosoles secundarios (Zafra, Peña y Álvarez, 2013). Si bien la contaminación atmosférica es un fenómeno que se presenta a escala microscópica, en muchas ocasiones es posible su detección a simple vista. Según el estado físico de las sustancias que contaminan, estas pueden clasificarse en partículas sólidas o líquidas; o bien en gases y vapores (IGME, 1999).

En nuestro caso de estudio, es posible visualizar que el componente ambiental atmosférico tuvo un valor máximo de -19, y según la tabla 2, la importancia del pasivo es irrelevante. Este resultado podría atribuirse principalmente al tamaño pequeño de la explotación. La presencia en el aire de material particulado liberado durante las voladuras es muy escaso, dado que estas se realizan exclusivamente para llevar a cabo la operación de recorte, las demás tareas extractivas son efectuadas mediante equipamiento en forma mecanizada (retroexcavadora y pala). La frecuencia de voladuras es discontinua, de todos modos, se estima que se realiza, como mucho, una vez por semana.

Otro factor que constituye el componente ambiental atmosférico es la afectación por ruidos. Estudios recientes señalan que hay una incidencia negativa en la salud de los operarios de empresas manufactureras que permanecen a diario cerca de las máquinas en las plantas de producción. Se estima que las personas expuestas a ruidos en niveles superiores a los 85 decibeles ponderados sufren perdida de la audición (Romero, Acero y Jaimes, 2016).

En nuestro caso de estudio, la principal fuente de emisión de ruidos está ubicada justamente en el interior de la planta de corte. Si bien no se pudo establecer una medida cuantitativa de los mismos, se vio que los operarios están expuestos a elevados niveles de ruidos. No obstante, a escasos metros de la planta, estos se atenúan considerablemente $y$ en principio no perturban a las poblaciones más cercanas. Por tal motivo, las acciones de mitigación y remediación no resultan prioritarias para este componente.

Tabla 2. Matriz de calificación cuantitativa de pasivos ambientales de la cantera La Represa en fase de explotación

\section{Cantera de piedra laja La Represa}

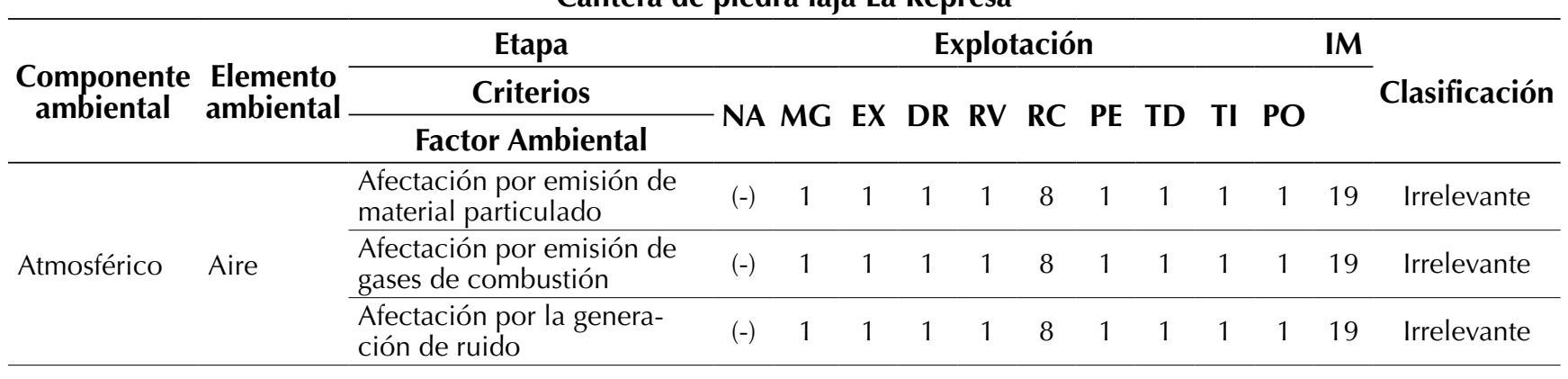




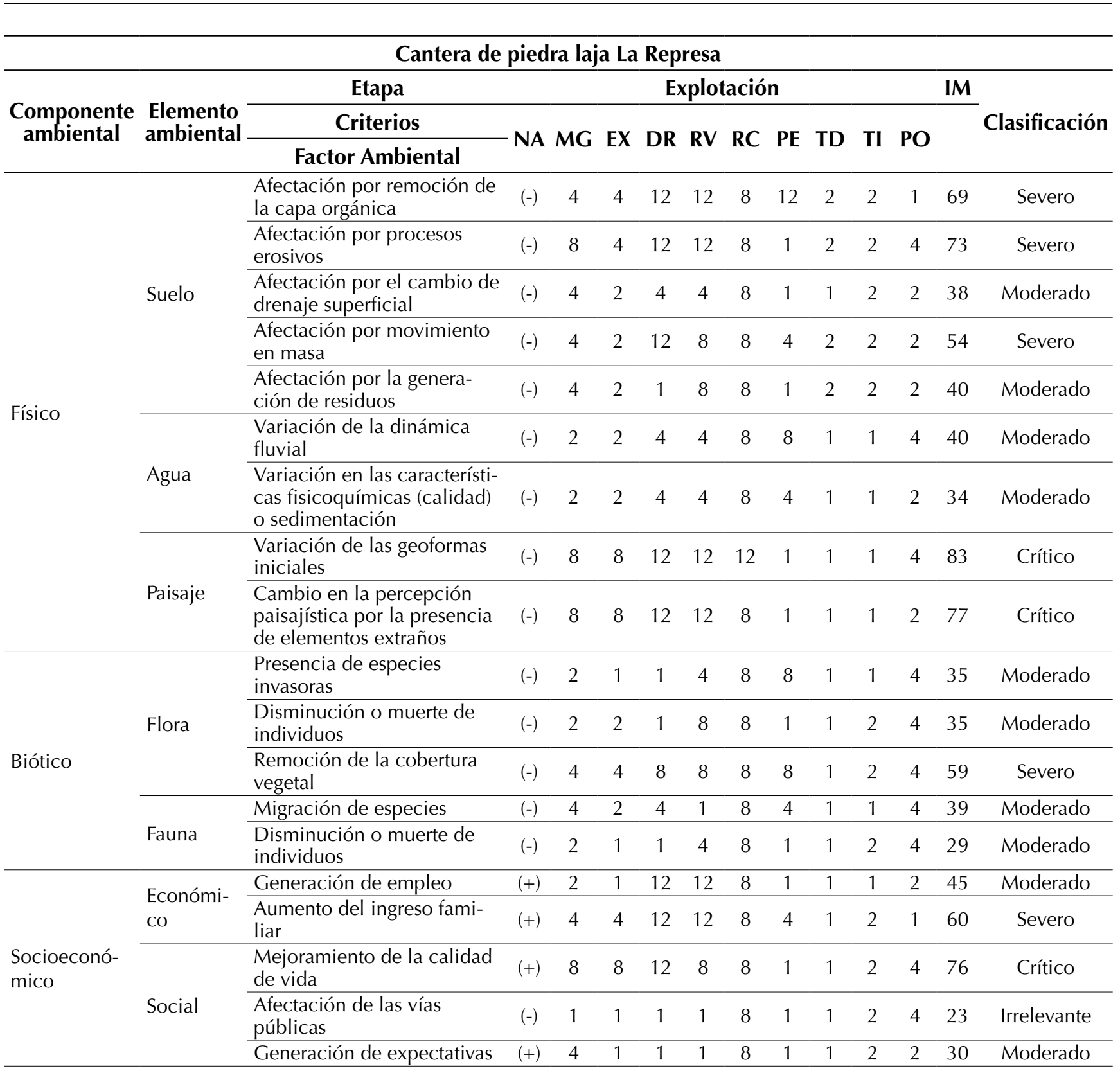

Fuente: elaboración propia.

\section{Componente físico}

\section{Elemento suelo}

La minería es una industria que produce un impacto negativo en la calidad de los suelos (Ballesteros et al., 2014). La degradación de este elemento puede causar la reducción de la función ecológica, debido a la alteración de ciertas características como el bajo contenido de materia orgánica, compactación, insuficiente profundidad, disminución de la resistencia a la erosión, entre otros (Chaudhuri, McDonald, Skousen y Pena-Yewtukhiw, 2015; Tizado y Núñez-Pérez, 2016).

Los resultados obtenidos para este elemento muestran valores dominantes entre -50 y -75 , lo que significa que los impactos son de importancia severa para la mayoría de los factores ambientales evaluados. La remoción de la capa superior del 
suelo y el arranque de las rocas son una afectación directa de las labores realizadas en una explotación a cielo abierto. Sin embargo, en la mayoría de los casos se recurre a efectuar la preservación de la cubierta orgánica del suelo durante esta etapa del ciclo extractivo.

La erosión del suelo se evidencia notablemente en toda el área de estudio, fenómeno que podría atribuirse principalmente a dos factores: por un lado, a la alteración del relieve; y por otro, al despojo de la cubierta vegetal causado por la explotación de estos recursos. Por lo descrito anteriormente, resulta necesario prever medidas tendientes a mitigar y restaurar este componente físico.

\section{Elemento agua}

La explotación minera afecta de forma moderada a este recurso. En el proceso industrial, el agua se utiliza únicamente en las plantas para refrigerar el elemento cortante (disco). En estos lugares se realiza el acabado de las piedras para su comercialización. El agua se compra y se transporta hasta las plantas en camiones cisternas, luego de ser utilizada pasa por un sistema constituido por piletas de decantación que permite la separación sólido-líquido; esta operación se realiza sin el agregado de reactivos químicos. Las aguas de rebalse (tratadas) son nuevamente utilizadas en la planta y los sólidos decantados son removidos cada un cierto tiempo. Se estima que cerca del $90 \%$ del agua es reutilizada en la planta y no se producen vertidos a cuerpos de aguas naturales.

El nivel piezométrico sufre alteraciones cuando se realizan operaciones extractivas en un lugar. Ante precipitaciones de intensidad inusual, las condiciones de inundación llegan a modificarse parcialmente, ya que las canteras actúan como receptores y amortiguadores locales (IGME, 1999). La acumulación de aguas (subterráneas) en los huecos producidos por la explotación ocurre frecuentemente; por lo que es necesario realizar maniobras (bombeo) tendientes a producir el desagote de estas, de los frentes de producción. En la figura 3 puede observarse la acumulación de agua en la cantera La Represa, cuando el ciclo de producción es interrumpido.

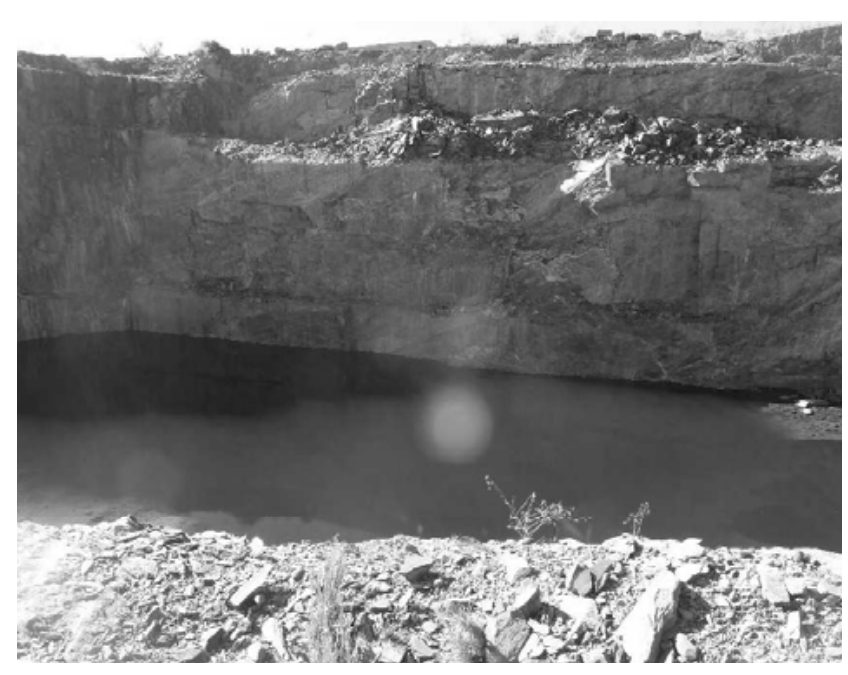

Figura 3. Acumulación de agua en la cantera La Represa cuando ocurre la interrupción de la operación

Fuente: elaboración propia.

\section{Elemento paisaje}

La explotación de la cantera de piedra laja produce un efecto negativo en el paisaje de importancia crítica en el lugar. La explotación se realiza en bancos de 25-30 m; estas alturas son ligeramente superiores a los recomendados por algunos países (15 m) en pos de la restauración y tratamiento de los taludes finales (IGME, 1999). No obstante, no existen urbes en las cercanías que puedan apreciar en forma directa dicha modificación paisajística. Se estima que el área afectada por la explotación es cercana a los $0,7 \mathrm{~km}^{2}$. Las escombreras, constituidas por valores próximos a 1.200.000 t, ocupan volúmenes próximos a $600.000 \mathrm{~m}^{3}$ (Ministerio de Industria, Comercio, Minería y Transporte, 2014), se han situado al oeste de las canteras en puntos cercanos a la ruta provincial No. 39, lugar observable desde las proximidades del murallón del dique La Florida. En este punto, es imperioso mencionar que La Florida es una localidad turística que suele ser muy concurrida en épocas estivales por ofrecer 
una amplia variedad de atractivos turísticos y recreativos. No obstante, la diferencia cromática observable entre el paisaje y las escombreras, desde dicho murallón, es relativamente poca; dado que las mismas se integran en cierta manera al entorno, debido a la escasez que existe en la cubierta vegetal del lugar. En general, las observaciones realizadas en este estudio son similares a las que habitualmente se describen para explotaciones a cielo abierto; donde las alteraciones más notables son aquellas producidas por los cambios en la fisonomía de la zona, generados por los huecos propios de la explotación y escombreras asociadas (IGME, 1999).

\section{Componente biótico}

\section{Elemento flora}

Este elemento de acuerdo a su IM es considerado una afectación severa. Un informe realizado por el Gobierno Provincial en 2014, da cuenta que en la zona que rodea al dique La Florida, se encuentran predominantemente pastizales y bosques serranos. La vegetación de la zona varía en función de la altitud, exposición y humedad disponible. En general se puede decir que en las quebradas la vegetación está compuesta, en el estrato superior, por especies arbóreas y arbustivas y en el estrato inferior por gramíneas y latifoliadas, situación común sobre los $850 \mathrm{~m}$ hasta los $1.300 \mathrm{~m}$ en las Sierras Centrales de San Luis (Ministerio de Medioambiente, 2014). Las tareas extractivas que se realizan han ocasionado la pérdida de gran parte de la flora autóctona en el área de trabajo, como se observa en la figura 2. Este aspecto deberá ser tenido en cuenta a futuro para Ilevar a cabo las tareas de restauración pertinentes.

\section{Elemento fauna}

La fauna del lugar probablemente emigró de la zona de las canteras por las tareas extractivas realizadas; en consecuencia, la afectación de este elemento es moderado. Según un informe realizado por el Gobierno de la Provincia de San Luis en
2014, los principales mamíferos en la zona son: laucha de campo (Calomys laucha), zorrino (Conepatus chinga), vizcacha (Lagostomus maximus), pichiciego (Chlamiphorus truncatus), iguana (Tupinambis sp.), iguanas (lagarto colorado y overo), mara (Dolichotis patagonum), cuis chico (Microcavia australis). Estudios realizados en la misma zona demuestran además la presencia de tuco-tuco (Ctenomys validus), cuis (Microcavia australis o Galea musteloides), hurón menor (Galactis cuja), gato del pajonal (Lynchailurus pajeros) e indicios de presencia de jabalí (Sus scrofa), especialmente en las laderas inferiores de las Sierras. Otras especies también detectadas son: ciervo colorado (Cervus elafus), perdices, liebre europea (Lepus cápense), armadillos y murciélagos (diferentes especies). En esta zona se han identificado mamíferos correspondientes a especies nativas y exóticas. Entre los reptiles se mencionan lagartijas de los géneros Liolaemus, Homonota y Phymaturus, matuastos (Diplolaemus, Leiosaurus y Pristidactylus), iguana colorada (Tupinambis rufecsens). Los ofidios se hacen presentes con la boa de las vizcacheras Boa contrictor occidentali, los colúbridos de los géneros Pseudoboa, Philodryas y Pseudotomodon y las víboras (Ministerio de Medioambiente, 2014).

\section{Componente socioeconómico}

Las principales actividades socioeconómicas de la zona son: ganadería, agricultura y minería, aunque en los últimos años la actividad turística desempeña un papel económico emergente en la región. Como parte de la ganadería, se desarrolla cría de bovinos, caprinos y porcinos y, en menor medida, equinos. Respecto a los cultivos se producen maíz, soja, alfalfa y sorgo, entre otros. A su vez la actividad minera se lleva a cabo principalmente en torno a los minerales de laja y mármol (Ministerio de Medioambiente, 2014).

No obstante, los 83 habitantes de la localidad de Riocito (Indec, 2010) viven casi exclusivamente de los réditos que la actividad minera; y en especial la extracción de piedra laja, les deja. De este 
modo, el impacto generado en el progreso socioeconómico de sus habitantes resulta positivo como crítico para este lugar.

\section{Conclusiones}

La información relevada en la zona de las canteras de piedra laja en la localidad de Riocito permitió concluir que los componentes ambientales con afectación crítica son el físico y el socioeconómico.

El elemento paisaje tuvo esta valoración debido a la modificación paisajística causada por la presencia de elementos extraños y la variación de las geoformas naturales del lugar. Cuando finalice la operación deberán realizarse trabajos tendientes a restaurar este componente ambiental. Sin embargo, es recomendable tomar medidas durante la operación, que puedan amortiguar dicha afectación. Una de ellas podría ser disminuir la altura de los bancos de explotación actuales (en algunos casos superan los $20 \mathrm{~m}$ ), esto facilitará el trabajo posterior de la atenuación de taludes.

En el presente trabajo se evidenció que la actividad minera en la zona tiene una afectación crítica positiva, dado que es una fuente económica primordial para el progreso socioeconómico de los habitantes de Riocito.

Los elementos ambientales suelo y flora tuvieron una afectación negativa severa. Al finalizar la explotación, el suelo primero deberá reacondicionarse para luego propiciar la revegetación del lugar. Entre las principales tareas se debería contemplar: realizar la integración de la morfología del terreno con el paisaje circundante, conseguir un buen drenaje, reducir o eliminar la alcalinidad o acidez de los suelos y descompactar el terreno para permitir el correcto enraizamiento de las especies vegetales. En cuanto a la flora, antes de realizar la revegetación del lugar será necesario establecer el futuro uso, o usos, que se les dará a estos terrenos. En general se seleccionan especies que puedan insertarse y subsistir según las condiciones propias del lugar.
Todas las tareas propuestas para el poscierre de la cantera deberán ser examinadas no solo desde un enfoque técnico sino también económico. Los elementos ambientales que posean afectaciones negativas críticas al finalizar las tareas extractivas deben considerarse prioritarias cuando se implementen los trabajos de remediación del lugar.

\section{Financiamiento}

El trabajo presentado se enmarca en el proyecto de investigación "Análisis medioambiental de operaciones mineras" (PROIPO 32416) de la Universidad Nacional de San Luis, Argentina.

\section{Referencias}

Ballesteros, M., Cañadas, E.M., Foronda, A., Peñas, J., Valle, F. y Lorite, J. (2014). Central role of bedding materials for gypsum-quarry restoration: An experimental planting of gypsophile species. Ecological Engineering, 70, 470-476.

Careddu, N. y Siotto, G. (2011). Promoting ecological sustainable planning for natural stone quarrying. The case of the Orosei Marble Producing Area in Eastern Sardinia. Resources Policy, 36(4), 304-314.

Chaudhuri, S., McDonald, L. M., Skousen, J. y PenaYewtukhiw, E.M. (2015). Soil organic carbon molecular properties: effects of time since reclamation in a mine soil chronosequence. Land Degradation \& Development, 26(3), 237-248.

Dolcemascolo, M. (2016). Estudio de pasivos ambientales en la provincia de San Luis para la generación de hormigones. (Tesis de grado). Carrera Ingeniería en Minas, Universidad Nacional de San Luis. San Luis, Argentina.

García U., C.A., García V., M.C., y Agudelo R., C.F. (2014). Evaluación y diagnóstico de pasivos ambientales mineros en la Cantera Villa Gloria en la localidad de Ciudad Bolívar, Bogotá DC. Tecnura, 18(42), 90-102.

Gómez, D. (1999). Evaluación del impacto ambiental. Barcelona, España: Editorial Aedos. 
Instituto Nacional de Estadísticas y Censos de la República Argentina (Indec) (2010). Base de datos REDATAM, cuestionario básico. Recuperado de https://redatam.indec.gob.ar/argbin/RpWebEngine.exe/PortalAction? \&MODE=MAIN\&BASE $=C P V$ 2010B\&MAIN=WebServerMain.inl

Instituto Tecnológico Geominero de España (IGME) (1999). Manual de restauración de terrenos y evaluación de impactos ambientales en minería. Vol. 2. Madrid: Ministerio de Industria y Energía.

Ley 24.585. Código de Minería de la República Argentina, Leyes Complementarias (1996). 6a. ed. Buenos Aires, Argentina.

Ministerio de Ambiente y Desarrollo Sustentable. (2016). Informe del estado del ambiente. Recuperado de https://www.argentina.gob.ar/sites/default/ files/mayds_informe_estado_ambiente_2016_ baja_1_0.pdf

Ministerio de Industria, Comercio, Minería y Transporte (2014). Plan Maestro de Minería 2014/2020.
Tratado de paz entre progreso y medio ambiente. San Luis, Argentina: Gobierno de la Provincia de San Luis.

Ministerio de Medioambiente (2014). Informe de manejo ambiental del Perilago La Florida. San Luis, Argentina: Gobierno de la Provincia de San Luis.

Romero D., G.A., Acero C., J. y Jaimes B, M. (2016). Generación de mapas de ruido (industrial) desde sistemas de información geográfica. Un acercamiento desde la literatura. Tecnura, 20(49), 152-166.

Tizado, E.J. y Núñez-Pérez, E. (2016). Terrestrial arthropods in the initial restoration stages of anthracite coal mine spoil heaps in northwestern Spain: potential usefulness of higher taxa as restoration indicators. Land Degradation \& Development, 27(4), 1131-1140.

Zafra M., C.A., Peña V., N.A. y Álvarez P., S. (2013). Contaminación por metales pesados en los sedimentos acumulados sobre el corredor vial Bogotá - Soacha. Tecnura, 17(37), 99-108.

\section{(c) $(1) \Theta(\Theta$}

